\title{
GPPS-BJ-2019-\#203
}

\section{Effects of Squealer Tip Geometry Deviation on Flow Field and Aerodynamic Performance Using Uncertainty Quantification Method and Sensitivity Analysis Method}

\author{
Wei Shi \\ Department of Energy and Power Engineering \\ shiwei15@mails.tsinghua.edu.cn \\ Beijing, China
}

\author{
Pingting Chen \\ Department of Energy and Power Engineering \\ cpt13@mails.tsinghua.edu.cn \\ Beijing, China
}

\author{
Xueying Li \\ Department of Energy and \\ Power Engineering \\ li_xy@tsinghua.edu.cn \\ Beijing, China
}

\author{
Jing Ren \\ Department of Energy and \\ Power Engineering \\ renj@tsinghua.edu.cn \\ Beijing,China
}

\author{
Hongde Jiang \\ Department of Energy and \\ Power Engineering \\ jianghd@mails.tsinghua.edu.cn \\ Beijing,China
}

\section{ABSTRACT}

The first stage rotor squealer tip is a key area in gas turbine both for aerodynamic performance and blade cooling tasks, which should be carefully designed. However, Harsh operating condition near the rotor squealer tip can cause the geometry of the squealer tip to degrade and manufacturing inaccuracies can also cause the squealer tip geometry deviate from the ideal design. These geometry changes from the ideal design would change the flow field near the blade tip, thus change the heat transfer performance and the aerodynamic performance much. Thus, it is important to quantitatively investigate the effects of squealer tip geometry deviation on flow field and aerodynamic performance.

In this paper, a typical transonic first-stage turbine is employed, and three important geometric features of squealer tip, the tip clearance height $(\mathrm{H})$, the squealer depth (D), and the squealer edge chamfer (R), are selected. An uncertainty quantification process is performed to study the effect of deviation of H, D and R on the flow field and aerodynamic performance. Many cases with different geometry features are checked in the current study using 3D RANS simulations. A parameter sensitivity analysis using Sobol's method is carried out to identify the key parameters for squealer tip aerodynamic performance.

The uncertainty quantification results show that the exists of the tip chamfer $(\mathrm{R})$ reduces the size of separation bubble and the dwelling range of the scraping vortex, thus, the blockage effect of the leakage flow is weakened, which results in larger amont of leakage flow and more mixing loss of squealer tips with edge chamfer than those without edge chamfer. The results of parameter sensitivity analysis show
\end{abstract}

that the height of tip clearance $(\mathrm{H})$ is the main factor which affect the aerodynamic performance of squealer tip. This work provides a certain guiding direction for the optimization design of the turbine groove tip.

\section{INTRODUCTION}

In order to meet the demand of high efficiency and high efficiency of gas turbine turbines, the inlet temperature of modern high performance turbines is rising, which increases the blade cooling tasks. In particular, the tip of the first stage rotor is directly exposed to the high temperature gas which makes the cooling tasks there tough. What's more, it is farthest from the rotating axis which makes it subjected to strong centrifugal force. Thus, the tip of the blade becomes the most ablated and failed zone [1][2]. On the other hand, the aerodynamic loss caused by the tip clearance accounts for about $1 / 3$ of the total loss inside the rotor channel[3]. Therefore, the tip of the turbine blade is a key area to maintain turbine reliability, durability and efficiency [4]. However, slight tip geometric deviation may influence much on the aerothermal performance of the blade tip. Thus, it is important to investigate the effects of squealer tip geometry deviations on flow field and aerodynamic performance.

Numerous studies with numerical and experimental method had been done on the influence of turbine tip geometry on the blade tip aerothermal performance [4]. Li et.al [5] investigated the typical squealer tip leakage flow characteristics on a high pressure turbine blade by CFD simulation. They found that most of the fluid entering the squealer cavity is from the leading edge region. Zou.et.al [6] 
studied the dominant flow structure near the squealer tip and its impact on turbine aerodynamic performance numerically. Gao et.al [7] reported the effect of tip clearance variations on aerothermal characteristics of a transonic turbine tip. Zhou.et.al [8] numerally studied the effect of squealer thickness, squealer height on the aerothermal performance of tip leakage flow. Fischer [9] and Ma et.al [10] analyzed the tip clearance flow field and heat transfer of the squealer tip by experimental measurement technique. These investigations indicate that geometry parameters influence much on the aerothermal performance of the blade tips. However, all of those researches paid attention to the 'idealized' squealer tip with sharp corners and fixed gap sizes.

In practice, the geometry of the squealer tip is not ideal but with deviations: harsh operating condition near the rotor squealer tip can cause the geometry of the squealer tip to degrade and manufacturing inaccuracies can also cause the squealer tip geometry deviate from the ideal design. These geometry changes from the ideal design would change the flow field near the blade tip, thus change the heat transfer performance and the aerodynamic performance much. Thus, it is important to quantitatively investigate the effects of squealer tip geometry deviation on flow field and aerodynamic performance.

In the last decade, non-intrusive probabilistic uncertainty method, such as polynomial chaos expansion (PCE) and probabilistic collocation had gradually attracted the attention of many researchers [11] by its ability to quantitatively investigate the effects of different factors. Montomoli et al [11] pointed out that the design process of the modern aircraft engine needed almost $90 \%$ CFD simulation study nowdays. The widespread use of computer simulation tools and its increasing capacity makes it possible to conduct uncertainty quantification (UQ) analysis in the gas turbine which requires large amount of CFD simulations. In addition, compared to the traditional Monte Carlo method, these UQ methods significantly reduced the simulation work. Montomoli et al. [12][13] used the Probabilistic Collocation Method (PCM) to study tip gap uncertainties in a multistage turbine and tip geometrical deviation with gaps and fillets. The results show that the effect of these small geometric variations on the prediction of component durability is higher than expected. They found that he small variation of the tip gap is a reason that causes the disagreement between CFD and experiments. Maesschalck et al. [14] investigated the effect of three geometrical variation of the blade-tip region due to manufacturing tolerances and profile degradation during engine operation on turbine performance with stochastic collocation method. The results confirm that it is necessary to consider the effect of these existing inherently and unavoidable tip geometry deviations in the early design phase of the turbine.

The current study presents an UQ analysis to quantify the effect of the squealer tip geometric deviations on the flow field and loss development in a typical transonic single-stage turbine passage with polynomial chaos expansion method. A parameter sensitivity analysis using Sobol's method is carried out to identify the key parameters for squealer tip aerodynamic performance. This work extensive the understand for the robustness of a squealer tip design by presenting the confidence intervals of the leakage flow amount and the aerodynamic performance.

\section{NUMERICAL METHOD AND VALIDATION}

\section{Description of Blade Geometric Model}

A typical transonic single stage turbine from the Institute of Thermal Turbomachinery and Machine Dynamics (TTM)[16], is selected to study the effect of geometric deviation of squealer tip on the flow field and aerodynamic performance. Figure 1 is the sketch of the TTM single stage turbine. The Mach number in the outlet is 1.0 and the Reynold number (based on the rotor exit velocity) is $1.6 \times 10^{6}$. The aerodynamic parameters of the TTM turbine are listed in Table 1 .

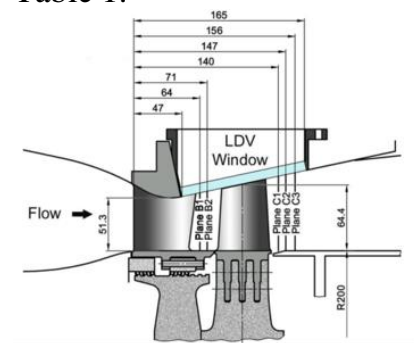

(a)Meridional flow path

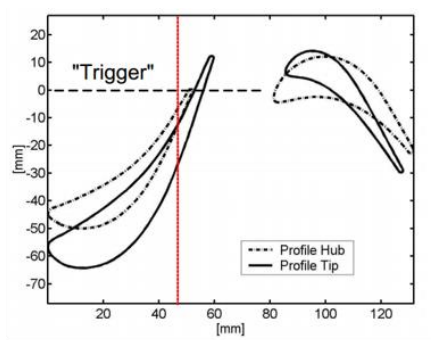

(b) Blade Profiles
Figure 1 Sketch of TTM single stage turbine [17] Table 1. TTM turbine specifications

\begin{tabular}{cc}
\hline \hline Parameter & Range \\
\hline Reynolds number, Re & $1.6 \times 10^{6}$ \\
Exit Relative Mach number & 1.0 \\
Rotor Blade count & 36 \\
Speed(rpm) & 11000 \\
Rotor pressure ratio & 3.12 \\
Inlet Total Temperature $(\mathrm{K})$ & 454.4 \\
Outlet Average pressure $(\mathrm{Pa})$ & 11078 \\
$\mathrm{~T}_{\text {tot,in }} / \mathrm{T}_{\mathrm{w}}$ & 1.4 \\
\hline
\end{tabular}

\section{Numerical Methodology}

Three-dimensional steady viscous Reynolds-averaged Navier-Stokes equations are solved to simulate turbine squealer tip geometric deviation in the UQ analysis. The commercial CFD code STAR-CCM+ is applied with shear stress transport (SST) turbulence model. The double precision and coupled solver are used in the study.

The full computational domain contains the stator and the rotor with squealer tip as shown in 0 (a). The stator domain is divided by the IGG module of the commercial software NUMECA and keeps unchanged for different rotor cases. Polyhedral mesh with prism layers around the walls is generated in STAR-CCM+ to keep $y+\sim 1$. The grid in the squealer tip is refined, and more than $70 \%$ of the grid nodes are distributed in the squealer tip area to ensure spatial resolution of the tip area.

Total pressure and total temperature are specified at the stator inlet based on the design operating condition, and static 
pressure are set at the rotor outlet. Both the surfaces at the circumferential side of the stator and rotor are specified as rotational periodic boundary conditions.

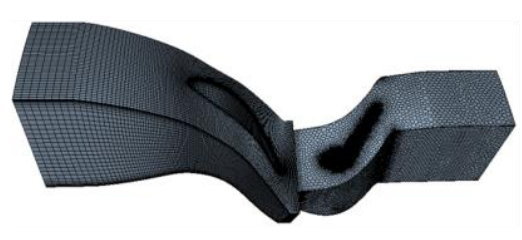

(a)

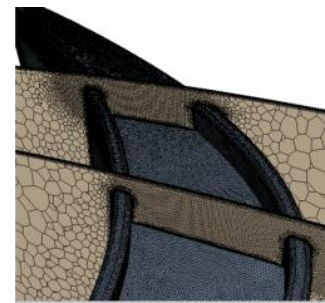

(b)
Figure 2 Computational domain and mesh (a) and zoom of the squealer tip(b)

Grid sensitive study is carried out to check the effects of grid resolution on the simulations. Different grid refinement strategies are presented and the total number nodes of the grids used in the grid sensitive study are 0.7 million, 1.08 million, 1.70 million, 2.88 million, 4.98 million and 7.88 million. The grid independent criterions are set as the changes of mass flow and the total pressure coefficient no more than $1 \%$ between the two cases. At the last, the 4.98 million grids are used to perform the numerical simulations. It is important to note that the total cell number differs with different geometry configurations studied here.

The CFD method validation is carried out in another transonic cascade used by Nicholson et al. in [18]. The Mach number is 0.96 and the Reynolds number of this cascade is $1.113 \times 10^{6}$, which is similar to the transonic cascade employed in the current study. Figure 4 shows the measured and simulated Mach number distribution in the mid-span of the blade. The simulated results are in good agreement with the experimental results, indicating that The CFD method modelled the aerodynamics of the cascade well.

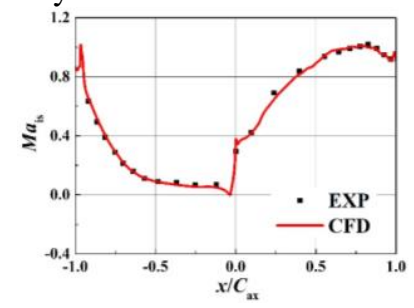

Figure 3 Numerical method Validation

\section{UNCERTAINTY QUANTIFICATION METHODOLOGY}

\section{Polynomial Chaos Expansion}

The uncertainty quantification (UQ) method used is the non-intrusive polynomial chaos expansion (NIPCE) in this study[11]. The non-intrusive method is based on some deterministic solutions to estimate the characteristics of random output variables. The statistical properties (mean, standard deviation, skewness, etc.) of the relevant parameters are obtained through statistical post-processing. This method can utilize the existing commercial solver without modifying its control equation, so it is easy to operate and has more applications. This method was first introduced by Wiener[19]. It used the Hermite orthogonal polynomial to build the PCE model. Then Xiu and Karniadakis [20] improved it and proposed a generalized polynomial chaos to deal with different distribution forms. The mathematical basis of the method is polynomial theory, which is equivalent to constructing a proxy model for random variables, and uncertainty analysis is performed on the proxy model. Strict mathematical proofs show that there are corresponding optimal orthogonal polynomial bases for different distribution forms and converge at exponential velocity.

The PCE model can be constructed by expanding the random output variable on the orthogonal polynomial basis, as follows:

$$
\begin{aligned}
& Y(\theta)=a_{0} \psi_{0}+\sum_{i_{1}=1}^{\infty} a_{i_{1}} \psi_{1}\left(\xi_{i_{1}}(\theta)\right)+\sum_{i_{1}=1}^{\infty} \sum_{i_{2}=1}^{i_{1}} a_{i_{i_{2}}} \psi_{2}\left(\xi_{i_{1}}(\theta), \xi_{i_{2}}(\theta)\right) . \\
& +\sum_{i_{1}=1}^{\infty} \sum_{i_{2}=1}^{i_{1}} \sum_{i_{3}=1}^{i_{2}} a_{i_{i} i_{3} i_{3}} \psi_{3}\left(\xi_{i_{1}}(\theta), \xi_{i_{2}}(\theta), \xi_{i_{3}}(\theta)\right)+\ldots
\end{aligned}
$$

Where $\theta$ represents the input random variable, representing the orthogonal polynomial basis.

The above formula can also be abbreviated into a common format:

$$
\hat{y}\left(\xi_{j}(\theta)\right)=\sum_{i=0}^{P} a_{i} \psi_{i}\left(\xi_{j}(\theta)\right) .
$$

The spectral analysis method based on the Galerkin projection method is used to solving the PCE coefficient. The method uses the properties of Galerkin projection and orthogonal polynomial to obtain the coefficients. If put both sides of equation (2) to respectively projecting each orthogonal polynomial base $\psi_{i}^{(\theta)}$, it can be obtained

$$
\left\langle\mathrm{y}\left(\psi_{j}(\theta)\right)\right\rangle=\left\langle\sum_{i=0}^{P} b_{i} \psi_{i}(\theta) \psi_{j}(\theta)\right\rangle,(j=0,1, \cdots, P) \text {. }
$$

According to the definition of inner product and the orthogonality of orthogonal polynomials, the following equation (4) can be got,

$$
\left\langle\psi_{i}(\theta) \psi_{j}(\theta)\right\rangle=\left\langle\psi_{i}^{2}(\theta)\right\rangle \delta_{i j} .
$$

Each coefficient in the PCE model can be solved from the equation (3), that is,

$$
b_{i}=E\left[\mathrm{y} \psi_{i}(\theta)\right] / E\left[\psi_{i}(\theta) \psi_{i}(\theta)\right],(i=0,1, \cdots, P) .
$$

$E\left[\psi_{i}(\theta) \psi_{i}(\theta)\right]$ which represents the expected value of the known polynomial is easy to obtain in this equation. $E\left[\mathrm{y} \psi_{i}(\theta)\right]$ can be solved by numerical simulation or Monte Carlo sampling method. The PCE model is built when each PCE coefficient is solved, then the statistical properties of output variables can be solved as the following equation,

$$
\begin{gathered}
\mu=\mathrm{a}_{0}(x) . \\
\sigma^{2}=\sum_{i=1}^{N_{p}-1}\left(a_{i}^{2} \psi_{i}^{2}\right) .
\end{gathered}
$$




\section{Description of stochastic samples}

Figure 4 plot the sketch of squealer tip geometry and the geometric parameters. Three widely used geometric features of squealer tip in the industry and research are selected as the stochastic parameters, which are the tip clearance height $(\mathrm{H})$, the squealer depth (D), and the squealer edge chamfer (R).
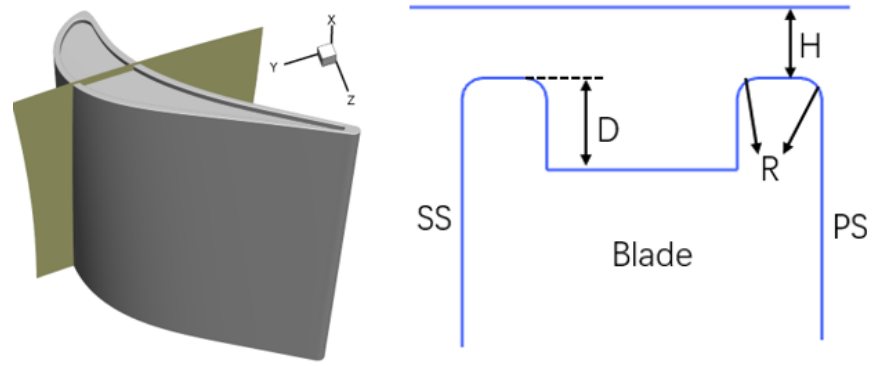

\section{Figure 4 Sketch of the squealer tip geometry and the geometric parameters}

These micro geometric variations existing inherently are caused by manufacture deviation and harsh operating conditions[14][24].These factors causing the geometry deviations from the ideal design to be in random distributions, which can be assumed to obey Gaussian distribution. However, the edge of squealer tip is generally sharp in the ideal design, but due to the harsh operation conditions, the sharp tip is easily to degrade, thus, the chamfer occurs and the chamfer is enlarged over time. Thus, the groove edge chamfers are assumed to be subjected to uniform distribution while the tip clearance and squealer depth are assumed to obey the Gaussian distribution. The variations and distribution of uncertainty parameters are shown in Table 2 . The tip clearance and squealer depth are the geometric deviations caused by the machining process, while the edge chamfering is caused by in-service degradation. Therefore, the UQ analysis is carried out with or without the presence of edge chamfering in this paper. Gaussian sample points are generated by PCE code combined with Sparse grid method when the edge chamfering is considered. The sample points are shown in the Figure 5. It's a two-dimensional UQ problem without considering the edge chamfering, and the full tensor method is directly used to generate the sample points.

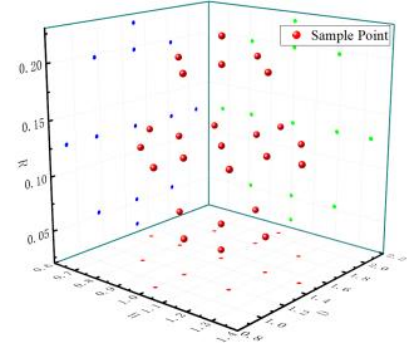

Figure 5 Sample points generated based on PCE + Sparse

Table 2. Variations and distribution of Uncertainty Parameters.

\begin{tabular}{|c|c|c|c|}
\hline Parameter & $\begin{array}{c}\text { Aver } \\
\text { age }\end{array}$ & Support & Distribution \\
\hline
\end{tabular}

\begin{tabular}{|c|c|c|c|}
\hline $\begin{array}{c}\text { Tip clearance } \\
\text { H(mm) }\end{array}$ & 1 & $(\mathbf{0 . 4 2 9 , 1 . 5 7 1 )}$ & $\begin{array}{c}\text { Gauss(Gauss- } \\
\text { Hermite) }\end{array}$ \\
\hline $\begin{array}{c}\text { Cavity Depth } \\
\text { D(mm) }\end{array}$ & 1.5 & $(0.5,2.5)$ & $\begin{array}{c}\text { Gauss(Gauss- } \\
\text { Hermite) }\end{array}$ \\
\hline $\begin{array}{c}\text { Corner Radius } \\
\text { R(mm) }\end{array}$ & $\sim$ & $(\mathbf{0 , 0 . 2 5 )}$ & $\begin{array}{c}\text { Uniform(Gau } \\
\text { ss-Legendre) }\end{array}$ \\
\hline
\end{tabular}

\section{Sobol's Sensitivity Analysis}

To identify the key parameters for aerodynamic performance of squealer tip, a parameter sensitivity analysis using Sobol's method is carried out. It is mainly used to analyze the contribution of each input random variable to the output response and the interaction of the input random variables. Since the PCE equation is very similar to the Sobol decomposition equation, the Sobol indices can be obtained using Gauss integral after obtaining the PCE expansion coefficient $S_{k}$, which is

$$
\mathrm{S}_{k}=\frac{\operatorname{Var}\left[\mathrm{y}\left(\psi_{j}(\theta)\right)\right]}{\operatorname{Var}[\mathrm{y}(\psi(\theta))]} \approx \frac{\sum_{k=I_{k}} \alpha_{k}^{2}}{\sum_{i=1}^{P} \alpha_{k}^{2}} .
$$

\section{RESULT AND DISCUSSION}

\section{Flow Field}

0 shows the leakage flow features of the conventional squealer tip. 7 sections along with the medial camber line are selected to analysis the flow field of squealer tip (S1-S7, as illustrated in Figure 6). These sections are colored by Mach number. Two-dimensional streamlines on each section and three-dimensional streamlines which pass through each section are also plotted. The blade surface is colored with local heat flux value. The leakage flow near the leading edge flows from the upstream edge to the squealer through the gap with the effect of the pressure gradient, and forms a scraping vortex with the effect of the downstream leakage jet and the moving casing. Some leakage flow that blocked by the scraping vortex will flow through it to the outlet gap of suction side, while the other will flow to the downstream part of the squealer tip. The leakage flow blocked by the scraping vortex also will impinge the bottom wall of the squealer tip, which will cause a significant increasing of the local heat flux. From the S3 section, the leakage flow which is at the tip region of suction side pass through the squealer tip and gradually forms a leakage vortex (the low velocity region near the suction side of squealer tip). When the leakage flow goes out of the gap, the strong momentum mixing with the mainstream will occur. And the mixing loss is an important component of the total leakage loss. 


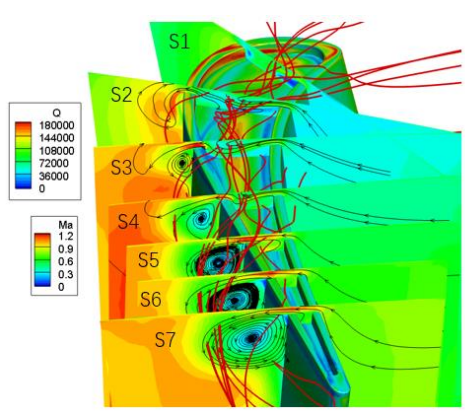

Figure 6 Flow diagram of the squealer tip

\section{Uncertainty Quantification Analysis}

With the existence of the chamfer at the edge of squealer tip, the definition of the exit position is somewhat complicated. In the current study, the center cross section of the squealer is selected as the gap exit position as shown in Figure 7.

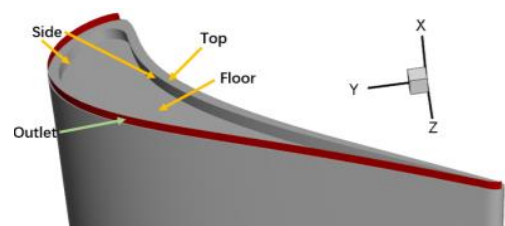

\section{Figure 7 Definition of the exit position and each wall}

Based on the mixing loss model proposed by Young and Wilcock[21], the mixing loss of the leakage flow and the mainstream can be expressed by the following formula:

$$
T \Delta s=\frac{m_{\text {leakage }}}{m_{\text {passage }}}\left[\left(V_{\mathrm{N}}-0\right)^{2}+\left(V_{\mathrm{ss}}-V_{\mathrm{T}}\right)^{2}\right] .
$$

According to the equation above, the mass flowrate of the leakage flow is the key parameter affecting the tip mixing loss. Wang. et al [22] also pointed out that the leakage flow can be used as an evaluation parameter for the aerodynamic performance of the tip. Figure 8. (a) shows the leakage flow deviation per unit length along the axial direction under the case that with and without the edge chamfer, respectively. The leakage flow increases firstly and reaches a local maximum value at the $50 \% \mathrm{Cax}$ and another local maximum point at $75 \%$ Cax. The geometric deviation has almost no effect on the tip leakage flow in the $0-15 \%$ Cax and $80 \%-100 \%$ Cax zone. However, the geometric deviations have a significant impact on the tip leakage flow in the 15\%-80\% Cax zone. In addition, the average value of the leakage flow with the edge chamfer is much larger than that without the edge chamfer. It indicates that as the increase of the wear degree of the squealer tip rib, the tip leakage flow enlarges. The accumulated leakage flow deviation along the axial direction is shown in Figure 8. (b). The mass flowrate of the accumulated leakage flow is normalized by the mainstream flowrate. It can also be seen that the influence of geometric parameter on the tip leakage flow is mainly in the $15 \%-80 \%$ Cax. The averaged tip accumulated leakage flowrate without the edge chamfer is $27.8 \%$ at the exit, while it is $30.4 \%$ with the edge chamfer.

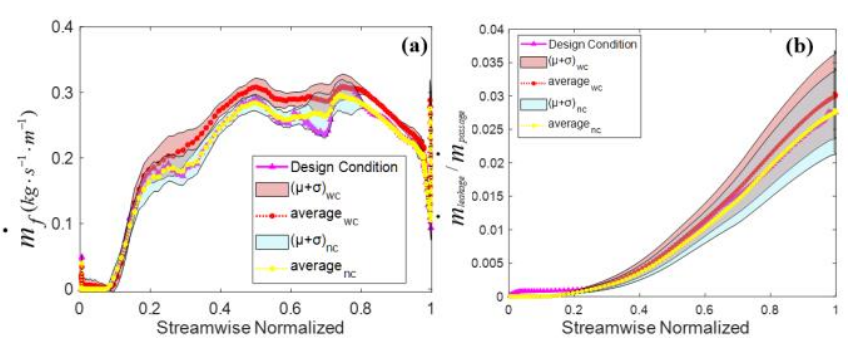

Figure 8 Distribution of leakage flow deviation along the streamwise direction

In order to analyze the difference of the leakage flow distribution between the chamfered and non-chamfered cases, Figure 9 presents the heat flux and relative Mach contour on the S1-S7 planes for the two cases. Significant change occurs on S1-S7 planes for the relative Mach number distributions with the presence of the edge chamfering, especially near the gap exit of the S3-S6 sections, which make the leakage flow increase in the $15 \%-80 \%$ Cax zone. As a result, the effect of rib chamfer on the flow structure in the squealer cavity is significant. The existence of edge chamfer reduces the separation bubble generated by the leakage flow at the rib top, and even eliminates the separation bubble. It will weaken the blocking effect of the squealer tip on the leakage flow, while the discharge coefficient will increase accordingly. Therefore, the Mach number and the outlet leakage flow at the gap exit will raise correspondingly. In addition, the position change of the high heat flux zone at the bottom of the squealer tip also indicates that the dwelling range of the scraping vortex in the squealer cavity will reduce and spread out of the gap in the middle chord. Therefore, the leakage flow doesn't impinge the bottom of the squealer tip strongly. So the leakage flow in the most zone of the squealer tip with the edge chamfer is higher than that without the edge chamfer.

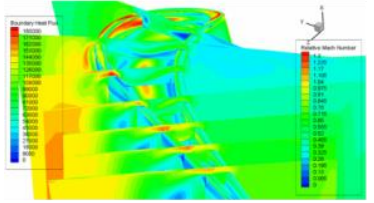

(a) Without chamfering

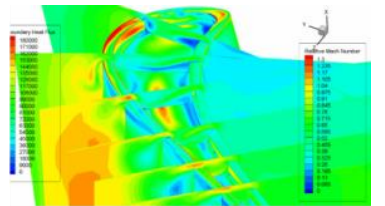

(b) With chamfering

\section{Figure 9 The flow field and heat flux contour of squealer tip}

To analyze the effect of the tip geometry variations on the aerodynamic loss in the blade passage, entropy generation is used to quantify the loss development. Metodi et.al[23] pointed out that entropy generation rate per unit volume is an effective tool to describe turbine losses and flow characteristics, especially in the case of film cooling of tip.

The local entropy generation is defined as:

$$
S_{v}=\frac{1}{T} \tau_{i k} \frac{\partial V_{i}}{\partial x_{K}}+\frac{\lambda}{T^{2}} \frac{\partial T}{\partial x_{i}} \frac{\partial T}{\partial x_{i}} .
$$

It contains two parts; one is viscous dissipation in the flow field, and another is thermal losses caused by irreversible thermal diffusion. The entropy generation is chosen as the former one in this paper. The local entropy generation is normalized by the case of the ideal design case. 
The Sv distribution of entropy generation along the streamwise direction are shown in 0 . It can be seen that the geometric deviation has little effect on the entropy generation of the blade passage at the $0-15 \%$ Cax and $80 \%-100 \%$ Cax zone. While the geometric deviation has a significant effect on the blade passage entropy generation at the 15\%-80\% Cax zone. The conclusion is basically consistent with the change of the leakage flowrate caused by the geometric deviation on the tip. The entropy generation loss in the blade passage have a positive correlation with the tip leakage flow as the equation (10) shown. The average of entropy generation without the edge chamfer is basically coincident with that of the design case. While the average entropy generation in the 20\%-80\% Cax zone with edge chamfer is higher than that without the presence of the edge chamfer. It indicates that the edge chamfer will increase the aerodynamic losses in the passage. The geometric deviation has the greatest influence on entropy generation in the $30 \%-60 \%$ Cax zone, and the maximum Std of entropy generation can reach $35 \%$ of the average.

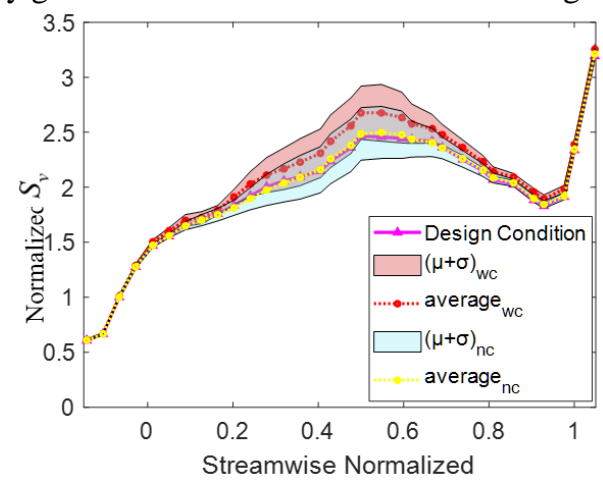

\section{Figure 10 Distribution of entropy generation deviation along the streamwise direction}

0 shows the normalized entropy generation contour at each axial section. The case that with the edge chamfer and without of that are both present in it. It can be seen that the entropy generation caused by the shear mixing, which is generated by the interaction of the leakage flow in the squealer cavity and the scraping vortex, is obvious. It is an important source of total entropy generation at each axial position. When the leakage flow spray out from the gap and is mixed with the main flow, the entropy generation increases inevitably. The high entropy generation zone in the main passage corresponds to the axial zone of the high leakage in Figure 8. Comparing the cases edge chamfer and without that, it can be seen that there is difference in the distribution of entropy generation of the squealer cavity and the main channel. The difference in the squealer cavity is attributed to the change of the vortex structure, especially the scraping vortex in the cavity. While the difference of the entropy generation in the main passage is caused by the difference of the distribution of the leakage flow. In addition, another high entropy generation region occurs near the trailing edge outside the passage. This is caused by the mixing between the rotor wake and the mainstream, which is corresponding to the rapid increase of the entropy generation in the downstream flow.

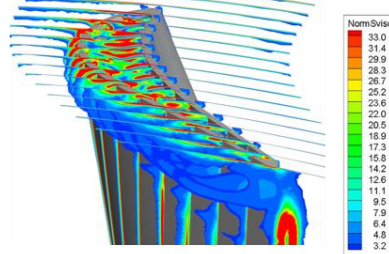

(a) Without chamfering

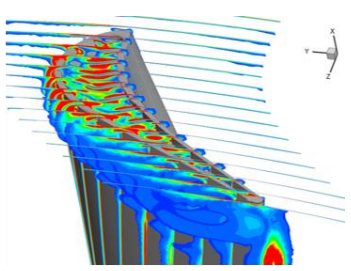

(b) With chamfering

\section{Figure 11 Distribution of entropy generation}

In order to assess the influence of the tip geometry deviation on the downstream flow field. Figure 12 shows the distribution of total pressure loss coefficient and the relative flow angle deviation along the radial direction at the 50\% Cax behind the TE.

The total pressure loss coefficient is defined as:

$$
\text { TPLC }=\frac{P_{t, \text { inlet }}-P_{t, \text { outlet }}}{P_{t, \text { inlet }}-P_{\text {outlet }}} \text {. }
$$

In which the $P_{t, \text { inlet }}$ is the mainstream inlet total pressure, $P_{t}$, outlet is the total pressure at the outlet. The static pressure at the CFD domain exit is $P_{\text {outlet }}$. The relative flow angle is defined as the angle between the flow direction and the frontal line. According to the influence of the geometrical deviation of the tip on the downstream flow field, the blade passage can be divided into four zones along the radial direction, $\mathrm{A}, \mathrm{B}, \mathrm{C}$ and $\mathrm{D}$, which represents from the hub to $10 \%$ span, $10 \%$ span$60 \%$ span, $60 \%$ span $-80 \%$ span and $80 \%$ span to the shroud, respectively. In the $\mathrm{A}$ zone, the total pressure loss coefficient at outlet is insensitive to the geometric deviation, while the relative flow angle will generate minimal change. However, the edge chamfer of the tip has no effect on the relative flow angel, because there is no apparent difference between them. In the B zone, there is a relatively small error bar for the total pressure loss coefficient and the relative flow angle at exit. In the $\mathrm{C}$ zone, the influence of the geometrical deviation of the tip with the edge chamfer on the total pressure loss coefficient and the relative flow angle is significantly different from that without the edge chamfer. The high loss in this zone are mainly caused by the passage vortex of casing. It can be seen in Figure 13 when the rib has edge chamfer, the passage vortex is stronger, and its position will move downward. In addition, the passage vortex and the leakage vortex are close when there is no edge chamfer. Therefore, the average of the total pressure loss coefficient at exit with the edge chamfer is higher than that without the edge chamfer in the $60 \%-70 \%$ span zone. While it is opposite in the $70 \%-80 \%$ span zone. It is obviously that the geometrical deviation of the tip mainly influences the D zone of the downstream flow field. The zone is mainly affected by the tip leakage vortex. It can be seen that the leakage vortex enhances and the total pressure loss increases when the squealer tip rib has the edge chamfer. The change of the relative flow angle will also be obvious, and the maximum deviation is $3 \mathrm{deg}$. 

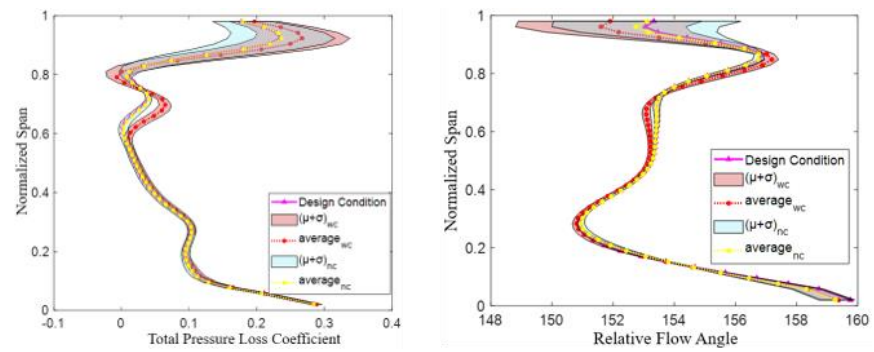

Figure 12 Distribution of Total Pressure Loss Coefficient and the relative flow angle deviation $50 \%$ Cax downstream

In order to analyze the difference of the distribution of the total pressure loss coefficient at the tip endwall of rotor outlet. The total pressure loss coefficient contour at the rotor outlet is shown in Figure 13. There are three distinct high pressure loss region along the radial direction in the passage, which are caused by the passage vortex of the hub, the passage vortex of the shroud and the leakage vortex of the squealer tip, respectively. Generally, the tip leakage vortex is the main source of loss. It is found that when the edge chamfer is present, the total pressure loss caused by the leakage vortex will increase significantly. And the total pressure loss caused by the passage vortex of the shroud also increases slightly. Therefore, the difference of the total pressure loss distribution at the tip endwall region in 0 is mainly due to the difference of the leakage loss under the different conditions that with and without the edge chamfer of the tip. It can be known from the 0 , the rib chamfer weakens the block effect of the leakage flow, which will increase the leakage flow of the tip outlet and enhance the mixing interaction of the leakage flow and the main-flow. Finally, it will result in a significant increase in the total pressure loss coefficient of the tip endwall region at the presence of the edge chamfer.

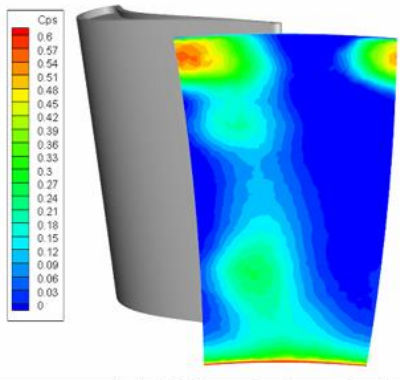

(a) Without chamfering

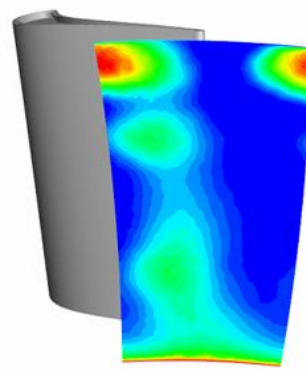

(b) With chamfering
Figure 13 Contour diagram of the total pressure loss coefficient of the rotor outlet with or without chamfering conditions

\section{Parameter Sensitivity Analysis}

To identify the key parameters for squealer tip aerodynamic performance, a parameter sensitivity analysis using Sobol's method is carried out as shown in Figure 14. It can be seen that as to the main effects, the most important factors affecting the cumulative tip leakage and turbine stage efficiency are the tip clearance height, which has a variance ratio of 0.989 and 0.978 , respectively. The influence of the squealer depth on the two aerodynamic indicators of the tip clearance is basically the same; The squealer edge chamfer has an increased effect on turbine stage efficiency.

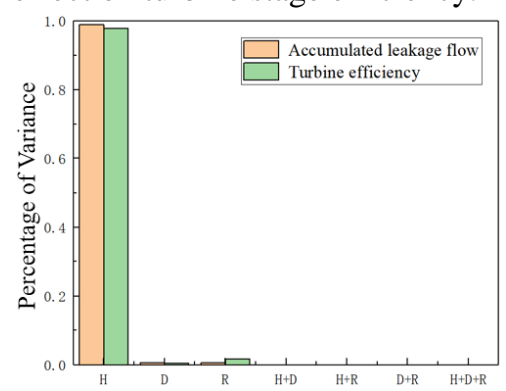

\section{Figure 14 Contribution of each parameter to aerodynamic performance deviation}

\section{CONCLUSION}

The present study investigates the effect of geometric deviation of squealer tip on the flow field and aerodynamic performance with three-dimensional numerical simulations. An UQ analysis is presented with the PCE model, integrated with Galerkin projection method and sparse grid method. The squealer with edge chamfer and without the edge chamfer are both considered in this paper. At the last, a Parameter Sensitivity Analysis is also performed using Sobol's method.

The tip chamfer reduces the size of separation bubble and the dwelling range of the scraping vortex, so that the blockage effect of the leakage flow is weakened, which results in the cumulative leakage and mixing loss of squealer tip with edge chamfer is higher than the cases without edge chamfer.

The height of tip clearance is the main factor which affect the aerodynamic performance of squealer tip. The interaction between the geometric elements is basically negligible.

It is crucial to consider the influence of the geometry change of the groove tip in the early stage of the optimization design of the turbine groove tip. This work provides a certain guiding direction for the optimization design of the turbine groove tip.

\section{NOMENCLATURE}

$\begin{array}{ll}H & \text { clearance height } \\ D & \text { squealer depth } \\ R & \text { the squealer edge chamfer } \\ H P T & \text { High Pressure Turbine } \\ P D F & \text { probability density function } \\ \text { Std } & \text { standare deviation }\end{array}$

\section{Greek symbols}

$\begin{array}{ll}\mu & \text { Mean level } \\ \sigma & \text { Standard deviation } \\ \theta & \text { input random variable } \\ \psi & \text { orthogonal polynomial basis }\end{array}$

\section{Subscripts \\ $1 \quad$ Blade row inlet \\ $2 \quad$ Blade row outlet}




\section{ACKNOWLEDGMENT}

This work is supported by Foundation 51706116 and 51621062. The author would like to appreciate the support of Shao Fei from China Aero Engine Research Institute for help on the blade geometry.

\section{REFERENCES}

[1] Bunker R S. A review of turbine blade tip heat transfer[J]. Annals of the New York Academy of sciences, 2001, 934(1): 64-79.

[2] ZHANG D H, ZENG M, WANG Q W. The Influence of Rotating Speed on Film Cooling Characteristics on GE-E3 Blade Tip with Different Tip Configurations. ASME Orlando FL USA 2009.

[3] Denton J D. Loss mechanisms in turbomachines[C]//ASME 1993 International Gas Turbine and Aeroengine Congress and Exposition. American Society of Mechanical Engineers, 1993: V002T14A001-V002T14A001.

[4] Bunker, R. S., “Axial Turbine Blade Tips: Function, Design, and Durability," Journal of Propulsion and Power, Vol. 22, No. 2, 2006, pp. 271-285.

[5] Li W, Jiang H, Zhang Q, et al. Squealer tip leakage flow characteristics in transonic condition[J]. Journal of Engineering for Gas Turbines and Power, 2014, 136(4): 042601.

[6] Zou Z.P., Shao F., Li Y.R., Zhang W.H., Berglund A. Dominant flow structure in the squealer tip gap and its impact on turbine aerodynamic performance[J]. Energy, 2017, 138: 167-184.

[7] Gao, J., Zheng, Q., Niu, X., \& Yue, G. (2016). Aerothermal characteristics of a transonic tip flow in a turbine cascade with tip clearance variations. Applied Thermal Engineering, 107, 271-283.

[8] Zhou C., Hodson H.P. Squealer geometry effects on aerothermal performance of tip leakage flow of cavity tips[J]. Journal of Propulsion and Power, 2012,28(3):556-567.

[9] Andreas Fischer, Jörg König, Lars Büttner, Jürgen Czarske,Clemens Rakenius, Heinz-Peter Schiffer, Tip clearance flow field measurements at a turbine rotor with squealers, 16th Int Symp on Applications of Laser Techniques to Fluid Mechanics, Lisbon, Portugal, 0912 July, 2012

[10] Ma, H., \& Wang, L. Experimental study of effects of tip geometry on the flow field in a turbine cascade passage. Journal of Thermal Science, 24(1), 1-9. (2015)

[11] Montomoli, Francesco, and M. Massini. "Uncertainty Quantification Applied to Gas Turbine Components." Uncertainty Quantification in Computational Fluid Dynamics and Aircraft Engines. Springer, Cham, 2019. 157-193.

[12] Montomoli F., Massini M., Salvadori S. Geometrical uncertainty in turbomachinery: Tip gap and fillet radius[J]. Computers and Fluids, 2011, 46(1):362-368.

[13] Mazzoni C M, Ahlfeld R, Rosic B, et al. Uncertainty quantification of leakages in a multistage simulation and comparison with experiments[J]. Journal of Fluids Engineering, 2018, 140(2): 021110.

[14] De Maesschalck C, Lacor C, Paniagua G, et al. Performance Robustness of Turbine Squealer Tip Designs due to Manufacturing and Engine Operation[J]. Journal of Propulsion and Power, 2017, 33(3): 740-749.

[15] Seshadri P, Parks G T, Shahpar S. Leakage uncertainties in compressors: The case of rotor 37[J]. Journal of Propulsion and Power, 2014, 31(1): 456466.

[16] Erhard, J. Design, construction and commissioning of a transonic test turbine facility[D], Graz. University of Technology,2000.

[17] Göttlich, E., Neumayer F., Woisetschläger J., and et al. Investigation of stator rotor interaction in a transonic turbine stage using laser doppler velocimentry and pneumatic probes $[\mathrm{J}]$, Journal of Turbomachinery, 2004, 126(2):297-305.

[18] Nicholson J.H., Forest A.E., Oldfield M.L.G., et al. Heat transfer optimized turbine rotor blades an experimental study using transient techniques[C]. ASME Paper, 82-GT-304, 1982.

[19] N. Wiener. The homogeneous chaos. Amer. J. Math., 60:897-936, 1938

[20] Xiu. Dongbin, and George Em Karniadakis. "The Wiener--Askey polynomial chaos for stochastic differential equations." SIAM journal on scientific computing 24.2 (2002): 619-644

[21] Young J B, Wilcock R C. Modelling the Air-Cooled Gas Turbine: Part 2-Coolant Flows and Losses[C]//ASME Turbo Expo 2001: Power for Land, Sea, and Air. American Society of Mechanical Engineers, 2001: V002T04A026-V002T04A026.

[22] Wang Y, Song Y, Yu J, et al. Effect of cooling injection on the leakage flow of a turbine cascade with honeycomb tip[J]. Applied Thermal Engineering, 2018, 133: 690-703

[23] Zlatinov M B, Tan C S, Montgomery M, et al. Turbine hub and shroud sealing flow loss mechanisms[J]. Journal of Turbomachinery, 2012, 134(6): 061027.

[24] Mischo, Bob Axial Turbine Rotor Aero-thermal Blade Tip Performance Improvement through Flow Control,2008, Doctoral Thesis 